\title{
Hospital de San Juan de Dios de Granada desde sus inicios
}

\section{San Juan de Dios Hospital in Granada: a review from its beginnings Hospital de São João de Deus de Granada desde os seus primórdios}

\author{
Jessica Medina García \\ Diplomada en Enfermería. Máster Nuevas Tendencias en Investigación en Ciencias de la Salud. Máster en Cuidados Integrales \\ de Enfermería en Procesos Nefrológicos. Becaria del X Programa Minerva de la Fundación Index. \\ Cómo citar este artículo en edición digital: Medina García, J. (2014) Hospital de San Juan de Dios de Granada desde sus ini- \\ cios. Cultura de los Cuidados (Edición digital) 18, 39. Disponible en: http://dx.doi.org/10.7184/cuid.2014.39.06> \\ Correspondencia: Calle Alcalá la Real no 100, Edf. Jaima, Bloque 11, Piso 2c, CP 18013,, Granada (Granada) \\ Correo electrónico: jmg.enfermera@gmail.com \\ Recibido: 25/11/2013; Aceptado: 15/02/2014
}

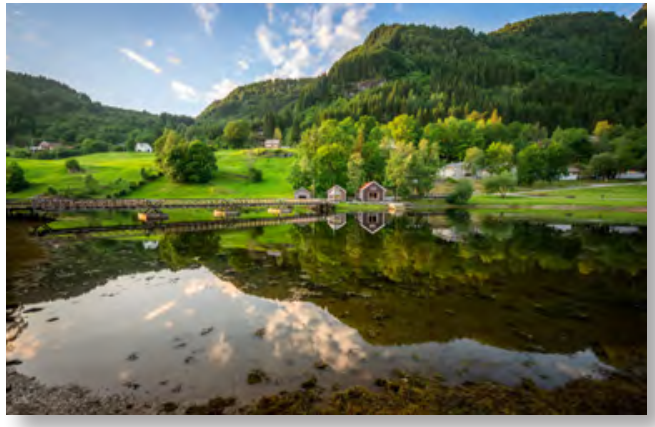

ABSTRACT

John of God was the epitome of hospitality and beneficent charity at that time. He practiced a humanistic Chistianity consolidated by his acts in hospital care, which he universalized with time. John Ciudad Duarte was the "seed" of what is now the Order of the Brothers Hospitallers, with 1.000 members of the religious order, 30.000 workers and 225 centres around the world. The material and methods which have been used include documents published from 1995 to 2013, thanks to an exhaustive bibliography of electronic resources and books. For this purpose, we have taken into account variable such as its opening, historical, context, the role of Juan Ciudad Duarte and the health system at that time. Among the results, we can mention that in the Hospital of San Juan de Dios, patients were put in a particular hospital according to their disease. Hygiene was of the utmost importance and patients' urine and excrements were properly disposed of. The way mentally ill people were treated was new. The work was carried out by own bothers of the order.

Key words: Hospital San Juan de Dios, Granada, History, Nursing.

\section{RESUMO}

Juan de Dios foi o epítome da hospitalidade e caridade beneficente do tempo que ele viveu. Realizou um humanista cristianismo consolidou suas ações na assistência hospitalar ao longo do tempo universalizado. Juan Cidade Duarte foi a "semente" do que é hoje a Ordem Hospitaleira, com 1.000 religiosos, 30.000 trabalhadores e 225 centros em todo o mundo. O objectivo geral do estudo é o de enquadrar o Hospital San Juan de Dios de Granada a partir do momento da sua fundação até hoje. Os materiais e métodos tem sido para recolher documentos publicados 19952013, através de uma pesquisa bibliográfica abrangente utilizando recursos eletrônicos e em papel na Universidade de Granada, tendo em conta variáveis como a data de abertura, o contexto histórico, uma figura de Juan Duarte 
de saúde da cidade e organização. Os resultados são mais proeminentes no hospital doente seleccionado os critérios de classificação, foi ótimo importancia da higiene e limpeza e que o tratamento dos doentes mentais era novidade. Cuidados de saúde é realizada por próprios irmãos de orden.

Palavras-chave: San Juan de Dios Hospital, Granada, Histótia, Enfermagem

\section{RESUMEN}

Juan de Dios fue el máximo exponente de la hospitalidad caritativa y benéfica de la época que le tocó vivir. Practicó un cristianismo humanístico consolidado por sus acciones en la asistencia hospitalaria que universalizó con el paso del tiempo. Juan Ciudad Duarte fue la "semilla” de lo que es hoy la Orden Hospitalaria, con 1.000 religiosos, 30.000 trabajadores y 225 centros repartidos por todo el mundo. El objetivo general del estudio es enmarcar el Hospital de San Juan de Dios de Granada desde el momento de su fundación hasta nuestros días. El material y método empleados ha consistido en recopilar documentación publicada desde el año 1995 al 2013, a través de una exhaustiva búsqueda bibliográfica mediante recurso electrónico y en formato papel en la Universidad de Granada, teniendo en cuenta variables como fecha de apertura, contexto histórico, figura de Juan Ciudad Duarte y asistencia y organización sanitarias. Los resultados más destacados son que en el hospital seleccionaba a los enfermos por criterios de clasificación, se le daba gran importancia a la higiene y al aseo, y un trato novedoso con los enfermos mentales. La asistencia sanitaria se llevaba a cabo por los propios hermanos de la orden.

Palabras clave: Hospital San Juan de Dios, Granada, Historia, Enfermería.

\section{INTRODUCCIÓN}

Esta institución hospitalaria fue fundada por San Juan de Dios allá en el año 1539, estando situada primero en la calle Lucena y posteriormente en la Cuesta de Gomérez (Larios Larios, 2004), trasladándose en 1554 a su ubicación actual en la calle de San Juan de Dios, dándole nombre a la vía. Es un hospital que a pesar de tener 470 años de historia, sigue vivo y continua desarrollando su labor asistencial. La mayoría de la literatura narra su historia hasta el siglo XIX, momento en el que los religiosos de San Juan de Dios se ven obligados a abandonar el hospital por la desamortización de Mendizábal, que produjo la exclaustración de la Orden Hospitalaria. Posteriormente pasó a ser gestionado por la Diputación Provincial de Granada. (Cambil, 2010)

El Hospital de San Juan de Dios destacó dentro de los hospitales granadinos del siglo XVI, ya que su fundación fue motivada por una manera de entender la caridad diferente a la acostumbrada para la época: el concepto medieval del buen morir. En el Hospital de San Juan de Dios se entendía la caridad hacia el prójimo en el sentido evangélico: "amarás al prójimo como a ti mismo". La cultura del cuidado vista desde la perspectiva cristiana altruistamente trasformó la sociedad y dio lugar al desarrollo de la Enfermería, a raíz de la cual surgió el cuidado de los enfermos y desvalidos (Fernandes de Freitas y Siles González, 2008). San Juan de Dios conoció de primera mano las necesidades de los enfermos, ya que intentó que los enfermos fueran cuidados con la mayor atención posible. Esto fue así porque él tuvo muy presente el trato inhumano que se le dio en el Hospital Real de Granada cuando estuvo internado, cosa que no quería que sucediera en su hospital. Allí se dio cuenta que era un error curar las enfermedades mentales con métodos de tortura (Fernandes de Freitas y Siles González, 2008). Para poder lle- 
var a cabo la iniciativa del nuevo hospital creó una infraestructura con la ayuda de sus seguidores que compartían su mismo pensamiento. Este hospital fue el único de toda Granada que no fue supervisado para llevar a cabo su labor, ya que los demás estaban en manos de una burocracia que controlaba la labor asistencial o bien por patronos que descuidaban su tarea. La excepción fueron los hospitales dirigidos por las cofradías, ya que la asistencia de los mismos se basaba en prestar servicio entre sus miembros a cambio de una prestación económica. El hospital estaba abierto a todo tipo de enfermos independientemente del sexo y enfermedad. Se seleccionaban según algunos criterios como clasificación por dolencias, en un periodo histórico en el que los enfermos eran colocados sin considerar su enfermedad (García Monlleó, 1996). Por otra parte, también tenían en cuenta a los enfermos infecciosos separándolos del resto, evitando así el contagio y las infecciones. A diferencia de otras instituciones del período, en el Hospital de San Juan de Dios, toda persona que necesitaba asistencia era recibida de una forma directa sin ningún requisito previo. Cuando Juan Ciudad Duarte vivía los recibía él mismo, y después en su ausencia siguieron realizado este quehacer sus colaboradores.

La hipótesis de trabajo consiste en el convencimiento de que el Hospital de San Juan de Dios ha desarrollado una serie de singularidades a lo largo de la historia que le ha permitido permanecer activo hasta nuestros días. Por tanto, el objetivo del estudio es enmarcar al Hospital de San Juan de Dios de Granada desde el momento de su fundación hasta nuestros días y determinar las peculiaridades del hospital que han permitido que éste perdure a lo largo de su historia.

\section{MATERIAL Y MÉTODO}

Esta revisión se ha llevado a cabo desde un punto de vista histórico, social y sanitario, me- diante una búsqueda bibliográfica mediante recursos electrónicos en bases de datos científicas de ciencias de la salud, considerando como principales fuentes CUIDEN, SCIELO y PubMed, además del catálogo de los fondos bibliográficos de la Universidad de Granada para la consulta de documentos en formato papel y tesis doctorales. El método ha consistido en recopilar documentación publicada desde el año 1995 al 2013, teniendo en cuenta consideraciones como fecha de apertura, contexto histórico, figura de Juan Ciudad Duarte, asistencia y organización sanitarias.

\section{RESULTADOS Y SU DISCUSIÓN}

La fundación de este hospital se inicia a finales de 1539, en el momento en el que Juan Ciudad comienza su dedicación en cuerpo y alma a asumir la causa de los necesitados y enfermos, a los cuales buscaba y recogía por las calles, llevándolos al principio a los zaguanes de la casa de los señores Venegas (Cambil Hernández, 2010). Eran tantos los que acudían a él que tuvo que alquilar un local en la calle Lucena y posteriormente se ubicó en la Cuesta de Gomérez (Larios Larios, 2004). En esta segunda no hay una clara referencia bibliográfica de cómo se adquirió pues Francisco de Castro (Castro, 1995) cita que "le compraron una casa para hospital" y sin embargo, otros autores como el padre de la Chica refiere que fue Juan quien la compró. Fuera como fuese, San Juan de Dios carecía de medios para permitirse la adquisición de una propiedad, por lo que las limosnas y el recaudo de dinero fueron cruciales para su obtención.

El obispo Don Sebastián Ramírez de Fuenleal, perteneciente a la Chancillería de Granada fue quien le impuso el hábito y el nombre Juan de Dios (Martínez Hernández, 1999). Este acto de reconocimiento público hizo que la economía del hospital comenzara a regularizarse, ya 
que numerosas personas pudientes colaboran con él y comienza a tener seguidores que actuaron como enfermeros (Cambil Hernández, 2010). De igual manera que sucedió en los anteriores hospitales, el de Cuesta de Gomérez se fue haciendo poco a poco insuficiente por la gran afluencia de enfermos que llegaban a él. Llegó un momento en el que las limosnas no eran suficientes para atender a los enfermos como Juan quería y el hospital tenía deudas (Larios Larios, 2004). En una de sus cartas él mismo afirmaba que no podía salir a la calle porque la gente a la que le debía dinero se le echaba encima reclamando lo que era suyo. Ante esta situación tuvo que recurrir hasta la más alta nobleza, teniendo incluso que viajar en 1547 a la Corte en Valladolid para hablar con el rey Felipe II, el cual le concedió grandes donaciones (Ventosa Esquinaldo, 2012). Sin embargo, San Juan de Dios con su carácter caritativo que tanto le caracterizaba, no supo llegar a Granada con tales donaciones, pues iba repartiendo por el camino hacia la ciudad todo lo recaudado entre los pobres que se encontraba por donde iba. Por tanto, el hospital seguía en la misma situación de ruina. A pesar de todo, los granadinos se volcaron dándole apoyo económico. Posteriormente, Juan de Ávila aconsejó a San Juan de Dios diciéndole que era conveniente buscar otro lugar con mayor capacidad para los enfermos, por lo que en 1554 el Hospital de San Juan de Dios quedó ubicado en su lugar actual (Laborde Vallverdú, 1981).

En el hospital se atendían a todo tipo de necesitados tales como exsoldados, huérfanos, viudas, mujeres públicas (Martín Casares, 1995), mendigos, peregrinos e incluso moriscos (García Pedraza, 1995) entre otros. El enfermo cuando ingresaba tenía que confesarse ante el rector, lo que indica la gran influencia que tenia la iglesia (García Montelleó, 1996). En el siglo XVI, el edificio contaba con 6 salas de enferme- ría y 25 camas. Era atendido por 25 religiosos, el Superior y la ayuda de una treintena de colaboradores, por lo que el personal del hospital era abundante y con una dedicación exclusiva de las tareas del mismo las 24 horas del día. Las medidas higiénicas eran equiparables a las que se llevan a cabo en los establecimientos sanitarios en la actualidad (Cambil Hernández, 2010), ya que entonces daban gran importancia a la higiene y al aseo, a la evacuación sistemática y correcta de las excreciones y orinas de los enfermos. Por otro lado, procuraban que los enfermos tuvieran cama individual y sábanas limpias, que los niños estuvieran separados de los adultos y de los pacientes infecciosos y que la asistencia estuviera distribuida con un orden a lo largo del día (comidas, medicinas y visitas médicas) (Ventosa Esquinaldo, 2012). Tras la muerte de San Juan de Dios, los Hermanos siguieron la labor de su antecesor teniendo solamente como guía las enseñanzas que este le había dado de palabra. Al carecer de normas escritas, los Hermanos fueron conscientes de la necesidad de dejar plasmado por escrito alguna serie de anotaciones como por ejemplo, un registro de las actividades diarias del hospital (Ventosa Esquinaldo, 2012). En éste se apuntaban los ingresos de los enfermos, su edad, fecha, situación en la que acudían al hospital, situación familiar, procedencia, y confesión entre otras. Por tanto, se puede considerar que San Juan de Dios comienza ya a prestar cuidados integrales: físicos, mentales, psicológicos, éticos, religiosos y sociales (Fernandes de Freitas y Siles González, 2008). Los reglamentos del Hospital de San Juan de Dios influenciaron la organización sanitaria española y en muchas otras partes del mundo (Fernandes de Freitas y Siles González, 2008).

San Juan de Dios fue el máximo prototipo de la hospitalidad caritativa y benéfica de la época que le tocó vivir. Practicó un cristianis- 
mo humanístico consolidado por sus acciones en la asistencia hospitalaria tales como la relación enfermo y cuidador, en una época donde la enfermedad era considerada como un pecado. Tal era esta creencia cultural que la gente llamaba primero al sacerdote y de forma secundaria llamaba al médico (Santos Oñate Tenorio y Santos Oñate Tenorio, 2013). San Juan Ciudad Duarte fue la "semilla" que dio fruto a lo que es hoy la Orden Hospitalaria, con más de 1.000 religiosos, 30.000 trabajadores y 225 centros repartidos por todo el mundo. San Juan de Dios se adelantó a su tiempo en cuanto al concepto de asistencia sanitaria y de organización sanitaria, y gracias a la labor de la orden este hospital ha llegado hasta nuestros días. Tras el deterioro del paso del tiempo, en la actualmente se está restaurando para poder proporcionar un servicio hospitalario de mayor calidad. Hoy por hoy, está especializado en la realización de cirugía médica ambulatoria. Presta otros servicios como consultas externas, radiología, hospitalización, área quirúrgica, hospital de día, terapia ocupacional y rehabilitación.

\section{CONCLUSIONES}

El Hospital de San Juan de Dios ha tenido cuatro ubicaciones: la casa de los señores Venegas, en la calle Lucena, en la calle Cuesta de Gomérez y en la calle de San Juan de Dios de Granada. Fue liderado por San Juan de Dios, quien se adelantó a su tiempo en cuanto al concepto de asistencia y de organización sanitarias. Esta es la clave esencial que le ha permitido seguir desarrollando la asistencia sanitaria a lo largo del tiempo. Al rededor de ésta, se agregan otros factores como son; ser el único hospital de la época exento de la supervisión por parte de la burocracia para realizar su asistencia, entender la caridad y ayuda para todos los enfermos por igual, tratar a los enfermos por sexos y según criterios de dolencias o posibles enfermedades contagiosas, además de estar dotado de un gran número de personal. Todo esto, ha permitido que el Hospital de San Juan de Dios, a pesar de contar con más de 470 años de historia, siga vivo y continúe desarrollando su labor asistencial.

\section{BIBLIOGRAFÍA}

- Cambil Hernández, M. E. (2010) Los hospitales de Granada: siglos XVI-XXI: tipología, catálogo e historia. Editorial Universidad de Granada,Granada.

- Castro, F. de. (1995) Historia de la vida y santas obras de San Juan de Dios y de la Institución de la Orden y Principios de su Hospital (ed. facs.). Cajasur, Córdoba.

- García Monlleó, F. J. (1996) La asistencia hospitalaria en Granada: El Hospital de San Juan de Dios (1552-1835). Universidad de Granada, Granada.

- García Pedraza. A. (1995) Una relación ignorada: Juan de Dios y los moriscos. Index de Enfermería. 14: 23-26.

- Fernandes de Freitas G. y Siles González J. (2008) Antropología y cuidados en el enfoque de San Juan de Dios. Index de Enfermería. 17(2): 144-148.

- Laborde Vallverdú, A. (1981) Seguirá Sonando La Campana: cronología del Hospital de San Juan de Dios de Granada. Años 1900 a 1980. Instituto Provincial de Estudios y Promoción Cultural, Granada.

- Larios Larios, J. M. (2004) El Hospital y la basílica de San Juan de Dios. Diputación de Granada, Granada.

- Martín Casares A. (1995) Cuidar descarriadas sanando su alma. Juan de Dios y las Prostitutas Granadinas del siglo XVI. Index de Enfermeria. 14: 27-30.

- Martínez Hernández, A. (1990) Historia del Hospital de San Juan de Dios: 1837-1937. Real Academia de Medicina y Cirugía, Murcia.

- Santos Oñate Tenorio M de los. y Oñate Tenorio A. (2013) San Juan de Dios, la implantación de una cultura de cuidados. Cultura de los Cuidados. 17(36): 44-49

- Ventosa Esquinaldo, F. (2012) Pensamiento de San Juan de Dios y la Orden Hospitalaria y su relación con la Enfermería: Conceptos y ValoresArchivo Museo San Juan de Dios Casa de los Pisa, Granada. 